\title{
Markarian survey and Markarian galaxies
}

\author{
A. M. Mickaelian * \\ NAS RA V. Ambartsumian Byurakan Astrophysical Observatory (BAO), Byurakan 0213, Aragatzotn province, Armenia
}

\begin{abstract}
Markarian survey (or the First Byurakan Survey, FBS) was the first systematic survey for active galaxies and was a new method for search for such objects. Until now, it is the largest objective prism survey of the sky $\left(17,000 \mathrm{deg}^{2}\right)$. It was carried out in 1965-1980 by B. E. Markarian and his colleagues and resulted in discovery of 1517 UV-excess (Markarian) galaxies. They contain many active galaxies, as well as powerful gamma-, X-ray, IR and radio sources (Mrk 180, 231, 421, 501, etc.), BCDGs (Mrk 116) and interacting/merging systems (Mrk 266, 273, etc.). They led to the classification of Seyfert galaxies into Sy1 and Sy2 and the definition of Starbursts (SB). Several catalogs of Markarian galaxies have been published (Bicay et al., 1995, Markarian et al., 1989, Mazzarella \& Balzano, 1986, Petrosian et al., 2007) and they are accessible in all corresponding databases. Markarian survey also served as a basis for search for UVX stellar objects (including QSOs and Seyferts), late-type stars and optical identification of IR sources. At present the survey is digitized and DFBS database is available. We review the main characteristics of the Markarian survey, its comparison with other similar surveys and the importance of Markarian galaxies in modern astrophysics.
\end{abstract}

Keywords: surveys - catalogues - techniques: spectroscopic-galaxies: active - galaxies: Seyfert-galaxies: Starburst - Virtual Observatory tools

\section{Introduction: Active Galaxies, AGN and their surveys}

The history of active galaxies goes back to 1943, when Carl Seyfert published a list of 8 galaxies with broad emission lines (Seyfert, 1943). Later on, radio galaxies were discovered (Bolton et al., 1949). In 1956 Guillermo Haro published a list of blue galaxies (Haro, 1956). Viktor Ambartsumian (1958) paid attention to some active processes and observing data connected with the central regions of some galaxies: blue/UV colors, emission lines, radio emission, outflows, etc. He predicted that more such objects should exist and new forms of activity may be found; this idea was in fact the very beginning of the unified scheme suggested much later by Antonucci \& Miller (1985). Similar discussions and direct indication on massive nuclei were given by Woltjer (1959). Predicted by Ambartsumian new types of active galaxies were the quasi-stellar objects (QSOs) discovered in 1963 (Schmidt, 1963) and the list of galaxies with anomalous colors (Markarian, 1963). To discover new such objects, find out what was their fraction and provide some statistics for further studies, Markarian conducted in 1965 a survey for UV-excess (UVX) galaxies. Some more types of active galactic nuclei (AGN) and other active galaxies were found in further works, such as BL Lac objects (Schmitt, 1968), Starburst (SB) galaxies (Weedman, 1977), LINERs (Heckman, 1980), etc.

The big variety of AGN types allows speaking about "AGN zoo", as all these types have certain peculiarities and need a reliable classification. First attempts to classify Seyferts were done in mid-1960s, when differences between NGC 4151 and NGC 1068 were noticed (prototypes of Sy1 and Sy2). Later on Weedman \& Khachikyan (1968) obtained the first spectra of Markarian galaxies and classified Seyferts into Sy 1/2 classes. Osterbrock (1981) introduced subclasses of Seyferts: 1.0, 1.2, 1.5, 1.8, 1.9 and 2.0. Later on Osterbrock \& Pogge (1985) found galaxies with Sy 1 features having narrow Balmer and other permitted lines, Narrow-Line Seyfert 1 (NLS1) galaxies; these objects also show strong FeII and soft X-ray. We use NLS also for other subtypes of Sy1 (NLS1.2, NLS1.5, etc.), as well as NLQSOs have been observed. For narrow line AGN (Sy2, LINER and SB), the classification is given by so called diagnostic or BPT (Baldwin, Phillips, Terlevich) diagrams (Baldwin et al., 1981, Veilleux \& Osterbrock, 1987). Anyway, due to the variety of their types and forms of activity, there is no final classification; very often classes refer to various properties, such as the morphology, optical spectrum, colour and/or spectral energy distribution (SED), radio loudness, polarization, etc.

*aregmick@yahoo.com 


\section{Markarian survey (First Byurakan Survey, FBS)}

In 1965, Markarian started the first observations of the First Byurakan Survey (FBS), which was aimed at covering the northern extragalactic sky by objective prism plates to search for UVX galaxies. The first list was published in 1967 (Markarian, 1967) and altogether 15 lists of 1500 galaxies were published by Markarian, Lipovetski and Stepanian. The selection of the low dispersion $(1800 \AA / \mathrm{mm}$ at $\mathrm{H} \gamma)$ provided a chance to follow SED and notice some broad (both emission and absorption) lines on one hand, and avoid overlaps on the other hand. Low-dispersion spectra cover the range 3400-6900 $\AA$, and there is a sensitivity gap near $5300 \AA$, dividing the spectra into red and blue parts. It is possible to compare these parts, easily distinguishing red and blue objects.

2050 Kodak IIAF, IIaF, IIF, and 103aF photographic plates in 1133 fields $\left(4^{\circ} \times 4^{\circ}\right.$ each, the size being $16 \mathrm{~cm} \times 16 \mathrm{~cm}$ ) have been taken. FBS covers 17,000 $\mathrm{deg}^{2}$ of all the Northern Sky and part of the Southern Sky at high galactic latitudes $\left(\mathrm{b}>15^{\circ}\right)$. The limiting magnitude on different plates changes in the range of $16.5^{m}-19.5^{m}$ in $\mathrm{V}$, however for the majority it is $17.5^{m}-18^{m}$. Each FBS plate contains low-dispersion spectra of some 15,000-20,000 objects, and there are some 20,000,000 objects in the whole survey.

We give in Table 1 the main observing and resulting characteristics of the FBS - Markarian survey. Though FBS spectra seem to be very similar, a thorough eye inspection with the help of $7^{\times}$lens provided opportunity to select peculiar spectra. To explain how difficult was the selection of peculiar spectra in the FBS, we give in Fig. 1 a standard FBS field and a collection of spectra of relatively rare interesting types of objects. Such spectra altogether are less than $5 \%$ among all in the FBS fields.

Table 1. Main observing and resulting characteristics of the FBS - Markarian survey.

\begin{tabular}{|l|l|}
\hline Items & Description \\
\hline Authors & B.E. Markarian, V.A. Lipovetskiy, J.A. Stepanian \\
\hline Years & $1965-1980$ \\
\hline Telescope & BAO $102 / 132 / 213 \mathrm{~cm}\left(40^{\prime \prime} / 52^{\prime \prime} / 84^{\prime \prime}\right)$ Schmidt \\
\hline Equipment & $1.5^{\circ}$ objective prism \\
\hline Emulsions & Eastman Kodak IIAF, IIaF, IIF, $103 \mathrm{aF}$ \\
\hline Plate size & $4.1^{\circ} \times 4.1^{\circ}, 16 \mathrm{~cm} \times 16 \mathrm{~cm}$ \\
\hline Spectral range & $3400-6900 \AA \AA$ with a sensitivity gap at $5300 \AA$ \\
\hline Dispersion & $1800 \AA / \mathrm{mm}^{\prime}$ at $\mathrm{H}_{\gamma}, 2500 \AA / \mathrm{mm}$ near $\mathrm{H}_{\beta}$ \\
\hline Scale & $96.8^{\prime \prime} / \mathrm{mm}^{\prime}$ \\
\hline Spatial resolution & $2.4^{\prime \prime}$ \\
\hline Limiting magnitude & $17.5^{m}-18.0^{m}$ in V \\
\hline Sky area & $\delta>-15^{\circ}$, all RA except the Milky Way $\left(|b|>15^{\circ}\right)$ \\
\hline Total coverage & 17,056 deg $^{2}$ \\
\hline Number of fields & $1139\left(\right.$ each 16 deg $\left.^{2}\right)$, distributed by 28 declination zones \\
\hline Number of plates & $1874($ at least one plate with m=17 in each field $)$ \\
\hline
\end{tabular}

Markarian survey was an outstanding study for all extragalactic (as well as galactic) astronomy; its main features may be given as:

- Markarian survey is the first systematic objective-prism survey,

- It is the largest objective-prism survey of the Northern sky $\left(17,000 \mathrm{deg}^{2}\right)$,

- It introduced a new method of search for active galaxies,

- Revelation of 1517 UVX galaxies: some 300 AGN and some 1000 HII galaxies, 


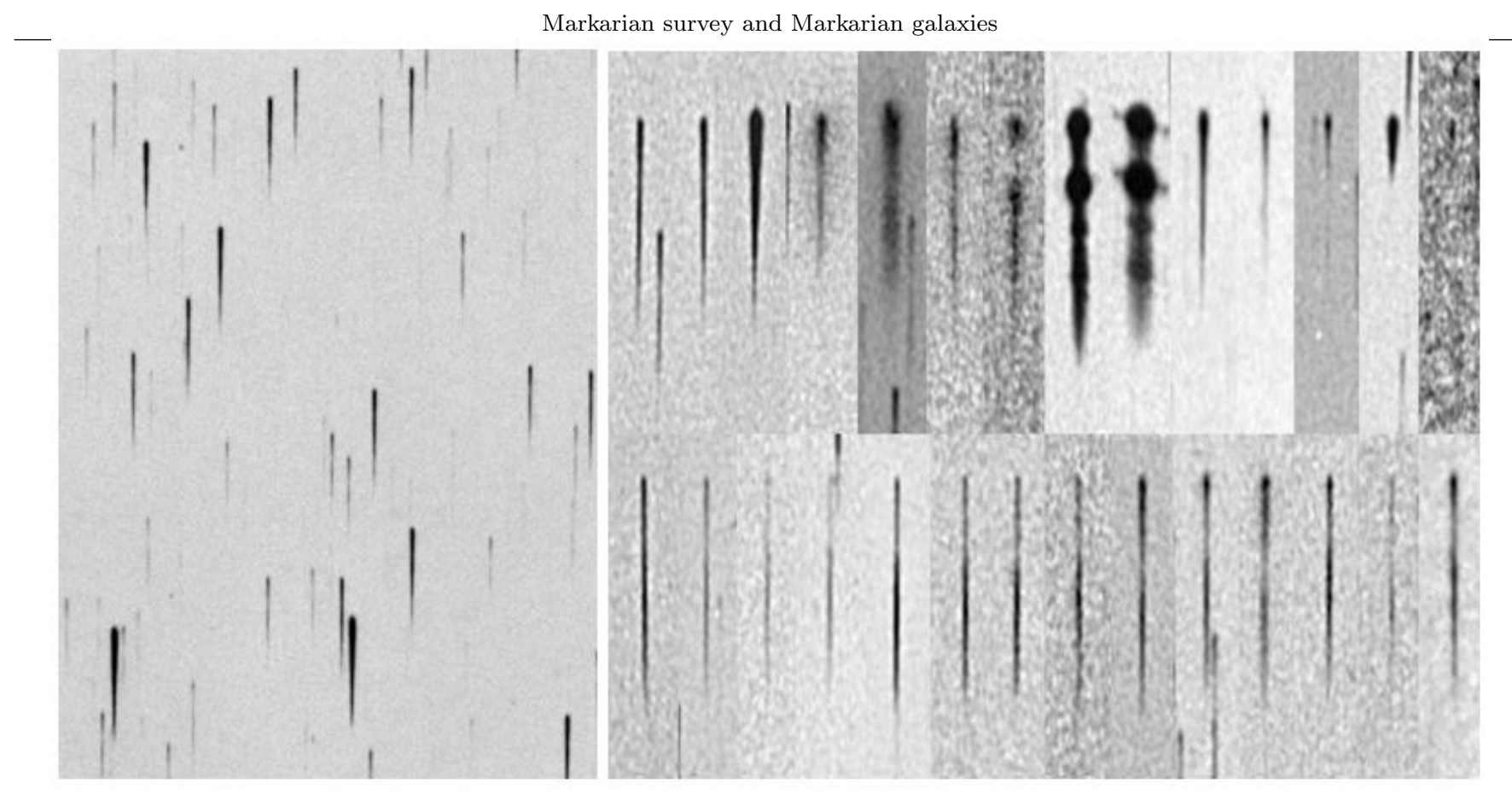

Figure 1. A standard FBS field showing similarity of most of the low-dispersion spectra and a collection of spectra of relatively rare interesting types of objects: Markarian galaxies, planetary nebulae, late-type stars (M and C), QSOs, CVs, white dwarfs and subdwarfs.

- Classification of Seyferts into Sy1 and Sy2 types (Weedman \& Khachikyan, 1968),

- Definition of Starburst (SB) galaxies (Weedman, 1977),

- Discovery of many new Blue Compact Dwarf Galaxies (BCDG),

- Revelation of 1103 FBS Blue Stellar Objects (BSOs; Mickaelian 2008) and 1471 Late-type Stars (Gigoyan et al., 2019),

- Optical identification of 1577 IRAS sources (samples of Byurakan-IRAS Galaxies (BIG; Mickaelian \& Sargsyan 2004) and Byurakan-IRAS Stars (BIS; Mickaelian \& Gigoyan 2006)); discovery of many new AGN and ULIRGs.

- Markarian survey led to many other objective prism surveys with better spectral resolution and deeper limiting magnitudes, including the Second Byurakan Survey (SBS, Markarian et al. 1983, Stepanian 2005).

\section{Markarian galaxies}

Markarian galaxies have nuclei with excessive amounts of ultraviolet emission compared with other galaxies (so-called UV-excess). So far, 1517 Markarian galaxies are known, as well as many more similar UVX galaxies exist. Markarian galaxies have been published in a series of 15 papers in Astrophysics (Astrofizika) and then listed in several catalogs. We give in Table 2 all major lists and catalogs of Markarian galaxies providing accurate positional, morphological, photometric, multiwavelength data and images.

Fig. 2 shows the distribution of Markarian galaxies on the celestial sphere by equatorial coordinates RA and DEC and Fig. 3 shows the distribution of various types of Markarian galaxies by Galactic coordinates $l I I$ and $b I I$.

Markarian galaxies have been studied by various observational methods, such as morphologically (e.g. Korovyakovskii et al. 1981), spectroscopically (Arakelyan et al. 1973, Markarian et al. 1988, Weedman \& Khachikyan 1968 and references therein), as well as in different wavelength ranges (see below). Petrosian et al. (1989) have studied double and multiple structure of some Markarian galaxies. Carone et al. (1996) received spectra for the Sy1 galaxy Mrk 509 and studied its optical continuum and emission-line variability. Petrosian \& Turatto (1986) investigated the relation of Markarian galaxies with Zwicky clusters. Santos-Lleó 
Table 2. Lists and catalogs of Markarian galaxies.

\begin{tabular}{|l|c|l|c|}
\hline Authors & Years & Description & Number of objs. \\
\hline Markarian et al. & $1967-1981$ & 15 original lists of galaxies with UV-excess & 1500 \\
\hline Kojoian et al. & $1978-1984$ & Accurate optical positions & 1500 \\
\hline Mazzarella \& Balzano & 1986 & The first catalog of Markarian galaxies & 1500 \\
\hline Markarian et al. & 1989 & $\begin{array}{l}\text { The First Byurakan Survey. A catalogue of } \\
\text { galaxies with UV-continuum }\end{array}$ & 1517 \\
\hline Bicay et al. & 1995 & $\begin{array}{l}\text { A multifrequency radio continuum } \\
\text { and IRAS faint source survey of Mrk galaxies }\end{array}$ & 899 \\
\hline Markarian et al. & 1997 & The FBS Catalogue of Markarian galaxies & 1517 \\
\hline Petrosian et al. & 2007 & $\begin{array}{l}\text { Markarian Galaxies. I. The Optical } \\
\text { Database and Atlas }\end{array}$ & 1544 \\
\hline
\end{tabular}

Table 3. Most important Markarian galaxies for various matters of extragalactic astronomy.

\section{Mrk galaxies}

Mrk 231

Mrk 421, 501

Mrk 116 (=IZw18)

Mrk 938

Mrk 110

Mrk 6

Mrk 926

Mrk 766

Mrk 273

Mrk 266

Mrk 231, 507

Mrk 530, 993, 1018

\section{Description}

the closest ULIRG, BAL QSO and most luminous IR galaxy in the Local Universe are among the highest known energy sources

the most metal-deficient (BCDG) (Mrk and SBS)

the first dynamic merger discovered observationally

intermediate between NLS1 and BLS1 $(\mathrm{FWHM}=4900 \mathrm{~km} / \mathrm{s})$;

understanding BLS1s and NLS1s differences

shows variations of spectral lines typical of different types of objects (Sy2 \& Sy1);

very high $\mathrm{H}$ column density in X-rays

one of the rare Sy1 galaxies having LINER properties

one of the most important NLS1 galaxies

a wonderful double-double nuclei galaxy

has a multiple structure nuclear region

super strongest FeII emitters (FeII $\lambda 4570 / \mathrm{H}_{\alpha}>2$ )

change their spectra from Sy1.9 to Sy1.0

et al. (2001) have carried out a monitoring of the optical and NIR spectrum and MIR imaging of the Sy 1 galaxy Mrk 279. Some Markarian galaxies have also been reported to have jets.

Markarian galaxies are rather important for various extragalactic studies, such as: Mrk 231 is the most luminous infrared galaxy (ULIRG) in the Local Universe, Mrk 116 is the most metal-deficient blue compact dwarf galaxy (BCDG) (most of the BCDGs are Mrk and SBS galaxies), Mrk 421 and 501 are among the most powerful sources, etc. We give in Table 3 some selected Markarian galaxies that are most important for various matters of extragalactic astronomy. Detailed studies of these and similar objects are given in other papers of this symposium.

Altogether 292 Markarian galaxies are present in the Catalog of QSOs and AGN (Véron-Cetty \& Véron, 2010) having activity types BLL, HPQ, QSO, Sy 1.0-1.2-1.5-1.8-1.9-2.0, NLS1, LINER, and HII. However, Markarian galaxies contain many more active ones, as not all have been classified for activity types. 

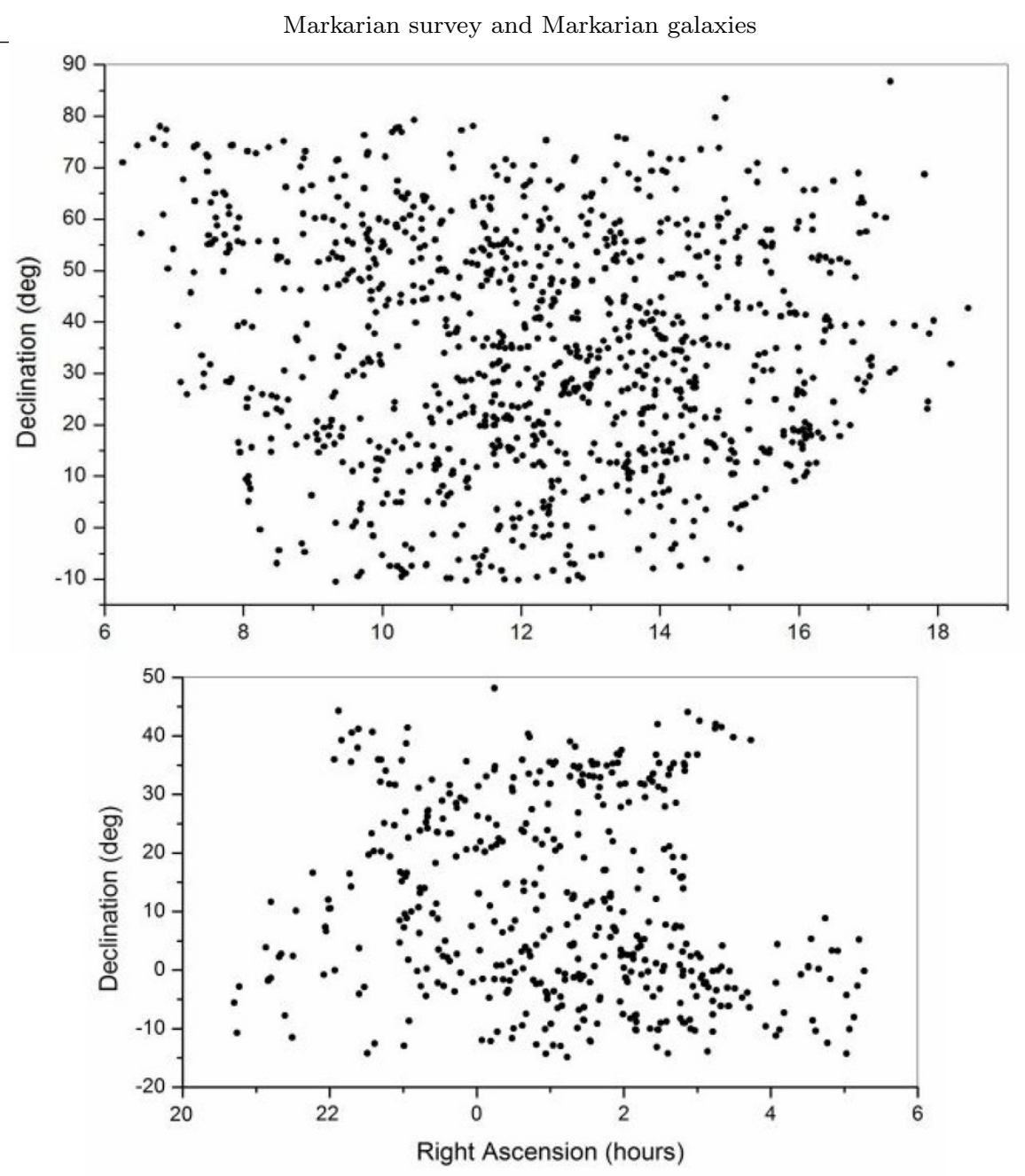

Figure 2. Distribution of Markarian galaxies on the celestial sphere by equatorial coordinates.

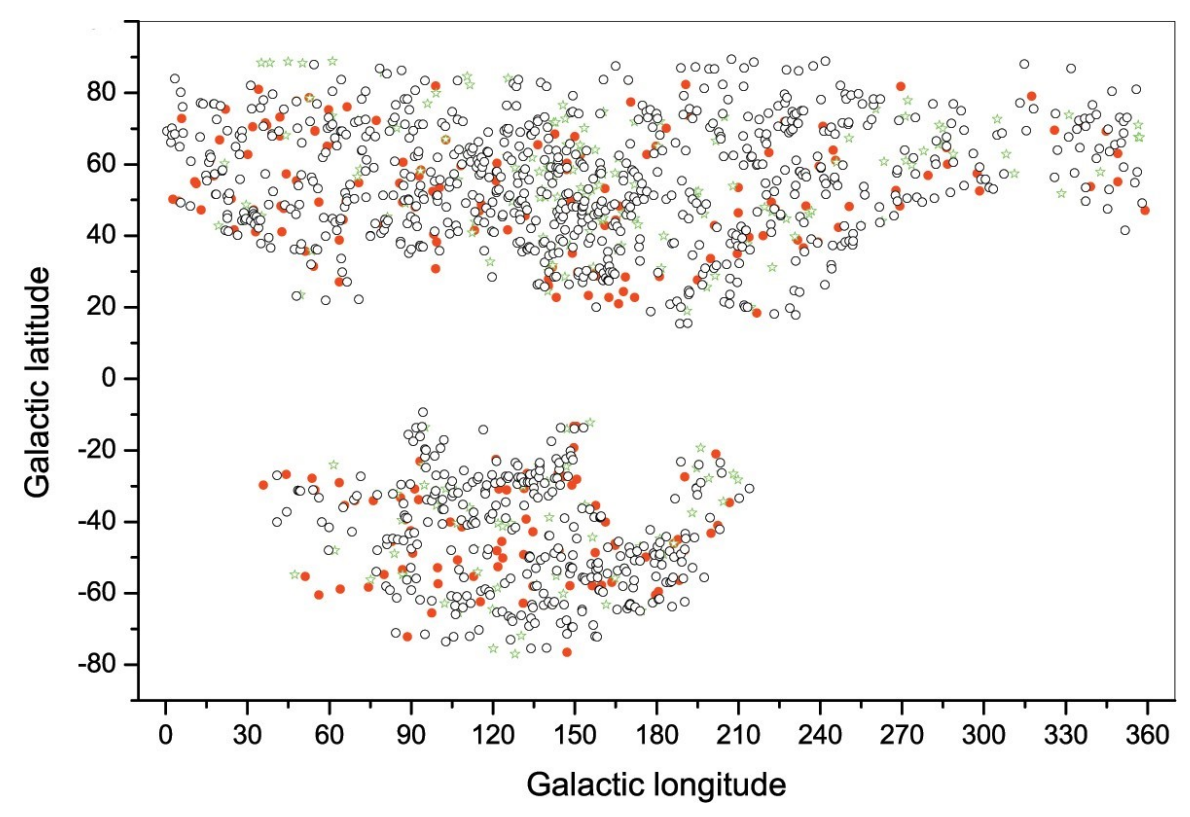

Figure 3. Distribution of Markarian galaxies on the celestial sphere by Galactic coordinates. Filled circles are AGN, stars are Starburst and HII galaxies, and open circles are galaxies without a sign of activity.

\section{Studies of Markarian galaxies}

Markarian galaxies have been observed in all wavelength ranges, from $\gamma$-ray to radio. E. g., they have been observed with the Arecibo radio telescope and for about $20 \%$ of them weak radio emission was detected (Tovmassian \& Terzian, 1974). Kojoian et al. (1976) have studied the radio spectra of Markarian galaxies. 
Kandalyan \& Petrosyan (1989) have studied Markarian galaxies as FIR sources. They are targets for all modern ground-based and space telescopes as well. E. g. the blazars Markarian 421 and 501 have been detected in all high- and very high energy surveys, such as ROSAT (Voges et al., 1999, 2000), ASCA (Ueda et al., 2005), BeppoSAX (Ciliegi et al., 2003), Chandra (Evans et al., 2010), XMM (2010), INTEGRAL (Bird et al., 2010), Fermi (Nolan et al., 2012), as well as observations by systems as the High-Energy Stereoscopic System (H.E.S.S.; Aharonian et al. 2005), MAGIC, the Very Energetic Radiation Imaging Telescope Array System (VERITAS; Arlen et al. 2013), Nuclear Spectroscopic Telescope Array (NuSTAR; Harrison et al. 2013), etc.

We have carried out studies of Markarian galaxies (Mickaelian et al., 2013), including their revised spectral classification based on the SDSS spectra (Ahumada et al., 2020), studies of their multiwavelength (MW) properties, etc. Examples of MW SEDs for three famous Markarian galaxies (Mrk 180, Mrk 231 and Mrk 421) are given in Fig. 4. These SEDs have been built and taken from the Italian Space Agency (ASI) Data Science Center (ASDC, http://tools.asdc.asi.it/SED/) using the ASDC SED builder tool.
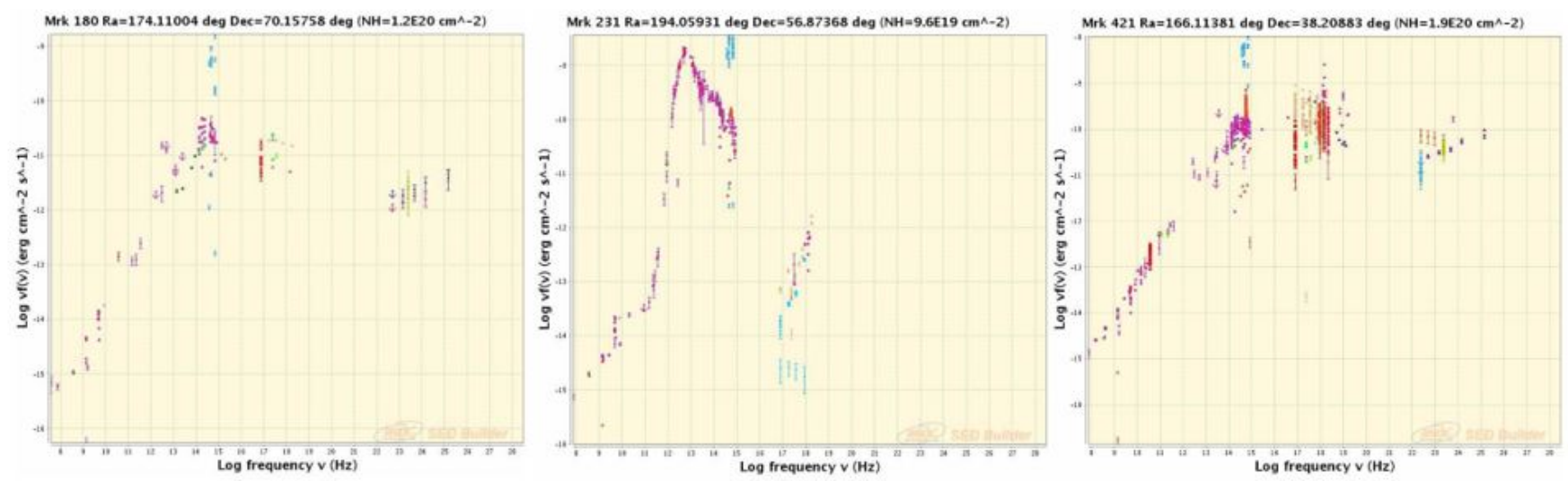

Figure 4. Spectral Energy Distribution (SED) of three famous Markarian galaxies: (from left to right) Mrk 180, Mrk 231 and Mrk 421.

Many more UVX and emission-line galaxies have been discovered in similar to Markarian surveys or by other studies. These are Arakelian galaxies having high surface bright- ness (Arakelian, 1975), Kazarian UVX galaxies (Kazarian et al., 2010), the University of Michigan emission-line galaxies (UM; MacAlpine \& Feldman 1982), Case Low-Dispersion Northern Sky Survey galaxies (CG; Pesch et al. 1991), the Montreal blue galaxies (Coziol et al., 1994), SBS UVX and emission-line galaxies (Stepanian, 2005), Kiso UV galaxies (KUG; Miyauchi-Isobe et al. 2010), Hamburg/SAO emission-line galaxies (Pustilnik et al., 2005), GALEX UV-luminous galaxies (Hoopes et al., 2007), etc.

\section{Digitized First Byurakan Survey - DFBS}

The Digitized First Byurakan Survey (DFBS; Massaro et al. 2008, Mickaelian et al. 2007) is the digitized version of the Markarian survey (or FBS). It is a collaborative effort of the Byurakan Astrophysical Observatory, Universita di Roma "La Sapienza" and MIGG s.r.l. (Italy), Cornell University (USA), and Hamburger Sternwarte (Germany). It included scanning of the plates, high accuracy (1" rms) astrometric solution, extraction software for images and spectra, photometric and wavelength calibration of the spectra, classification, creation of DFBS catalog and database, construction of user interface and webpage. Later on, the Armenian Institute of Informatics and Automation Problems (IIAP) also joined the project to reproduce the DFBS database in Armenia in frame of the Armenian VO project. 1874 FBS plates have been scanned. We give in Table 4 the main scanning and resulting characteristics of the DFBS.

Fig. 5 shows a fragment of "bSpec" - DFBS spectra extraction and analysis dedicated software written by Giuseppe Cirimele.

For the classification, templates for main types of objects discovered from FBS have been used; UVX galaxies, QSOs, white dwarfs, subdwarfs, cataclysmic variables, car- bon stars, as well as stars of all spectral types (from $\mathrm{O}$ to $\mathrm{M}$ ). The DFBS database is presently stored on a dedicated PC at Astronomical Observatory of Trieste (Italy) and can be accessed through web interface (http://www.ia2-byurakan.oats.inaf.it/). 
Table 4. Main scanning and resulting characteristics of the DFBS.

\begin{tabular}{|l|l|}
\hline Items & Description \\
\hline Teams & Byurakan Astrophys. Obs., Univ. Roma "La Sapienza", Cornell Univ. \\
\hline Years & $2002-2007$ \\
\hline Instrument & Epson Expression 1680 Pro scanner \\
\hline Scanning options & 1600 dpi $(15.875 \mu$ pix size $), 16$ bit, transparency mode, "scanfits" \\
\hline Plate size & $9601 \times 9601$ pix, $176 \mathrm{MB}$ file \\
\hline Spectra & $107 \times 5$ pix $(1700 \mu \mathrm{m}$ in length $)$ \\
\hline Dispersion & $33 \AA /$ pix average $(22-60 \AA /$ pix $), 28.5$ at $\mathrm{H} \gamma$ \\
\hline Spectral resolution & $50 \AA($ average $)$ \\
\hline Astrometric solution & $1 "$ rms accuracy \\
\hline Scale & $1.542 " /$ pix \\
\hline Photometry & $0.3^{m}$ accuracy \\
\hline Data volume & 1874 plates, $\sim 400 \mathrm{~GB}$ \\
\hline Number of objects & $\sim 20,000,000(\sim 40,000,000$ spectra $)$ \\
\hline
\end{tabular}

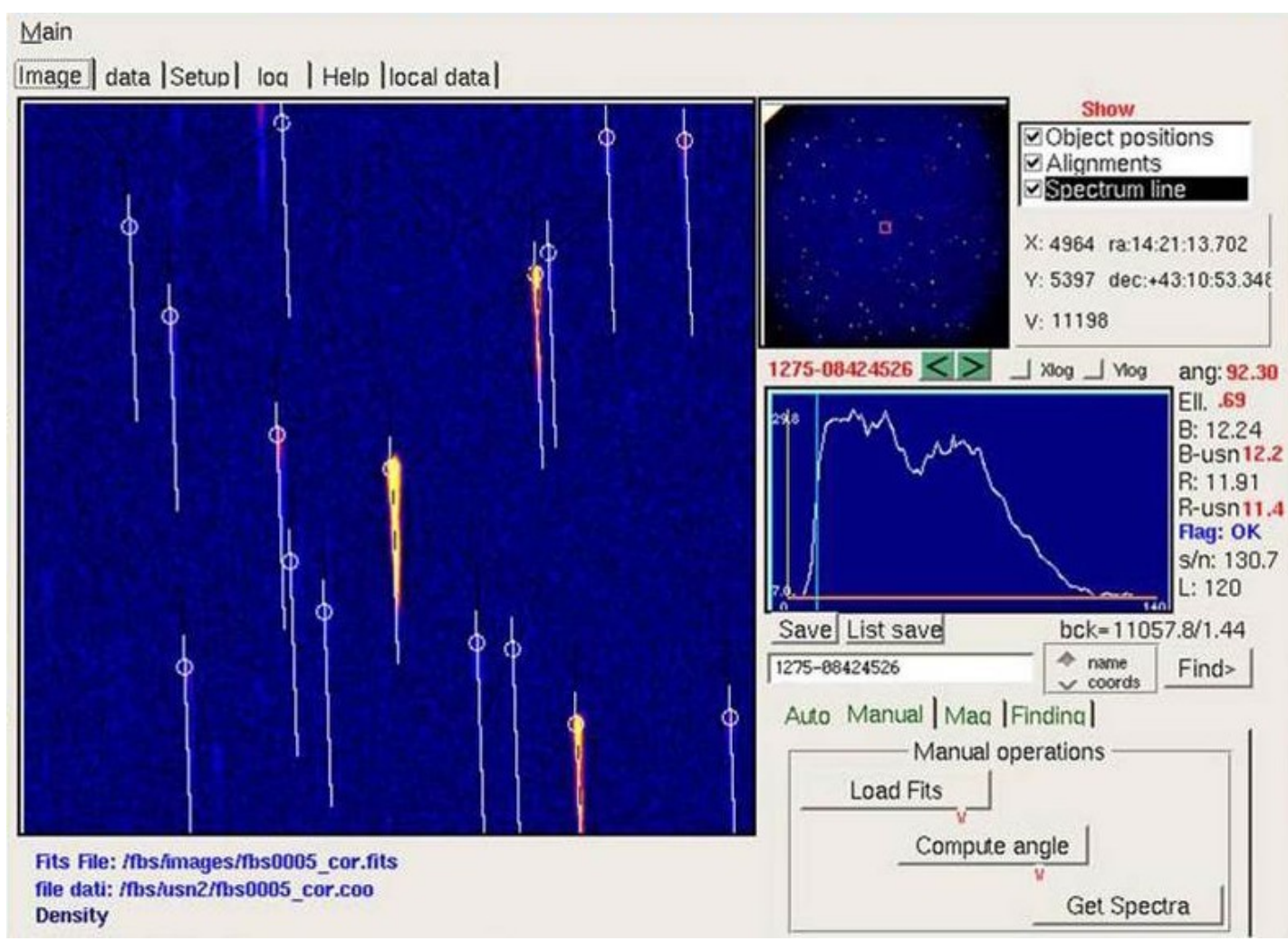

Figure 5. bSpec - DFBS spectra extraction and analysis software.

The user interface (the DFBS web portal) provides access to general information on the FBS and DFBS. It presently allows the following operations:

1) DFBS sky coverage,

2) Plate list,

3) Explore, allowing the display of a portion of plate around a given central RA, DEC position, interactive 
selection of one or more spectra, their collection and downloading,

4) Get Image, allowing users to select a portion of a plate in FITS format and all the spectra of this portion present in the database for downloading, as well as downloading of the whole selected field,

5) Get Spectra, allowing downloading all the spectra in the database within a given distance from a selected central position (cone search). Fig. 6 shows two webshots from the DFBS web interface: modes "Explore" and "Get Spectra".

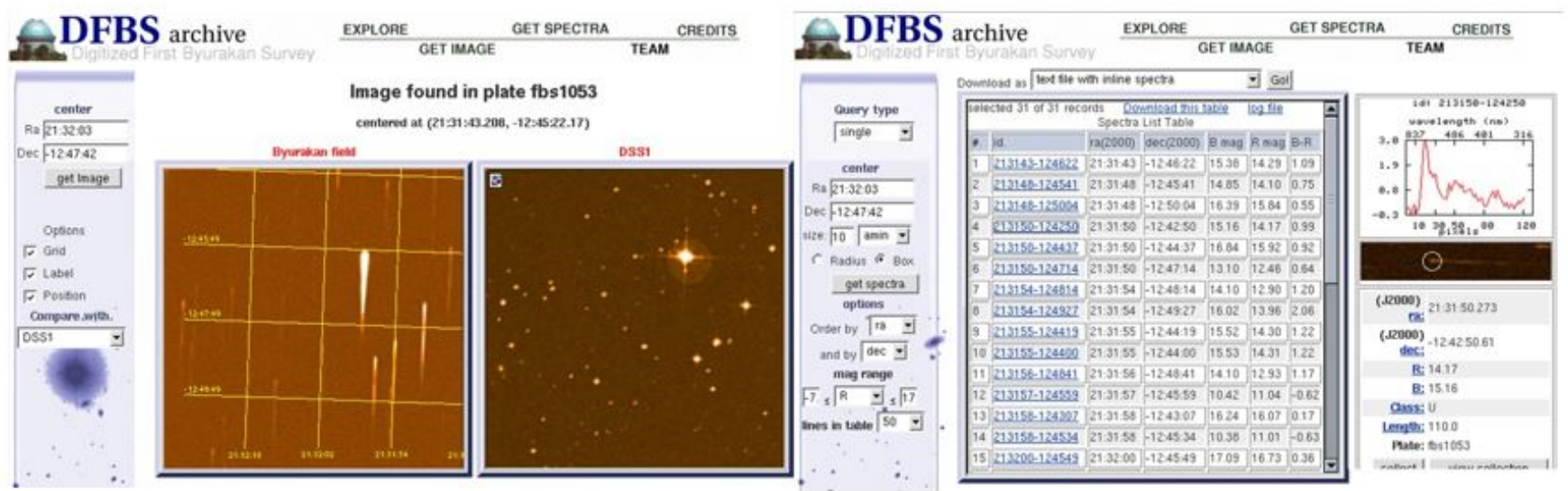

Figure 6. DFBS web interface: "Explore" and "Get Spectra" modes.

\section{Summary and Conclusions}

Markarian survey was the first systematic search for active galaxies, and Markarian galaxies led to discovery of many new AGN, spectral classification of Seyfert galaxies and definition of a new class of active galaxies, Starburst ones. Until now, Markarian survey is the largest area spectroscopic survey and the DFBS contains the largest number of spectra (some 20,000,000 objects).

We give in Table 5 a comparison of the main characteristics of large spectroscopic surveys. The first five surveys are objective prism ones and have been done using Kodak emulsions and only SDSS has been done with CCD using $u, g, r, i$, and $z$ filters. In all cases, the most important goals were to discover active galaxies, as well as SDSS also has carried out the largest ever galaxy redshift survey (4.5 million objects). Such surveys are also an ideal tool for optical identifications of X-ray, IR, and radio sources; such projects have been carried out using FBS (Mickaelian, 1995) and HQS (Mickaelian et al., 2006, Zickgraf et al., 2003).

Markarian survey led to the discovery of 1517 UVX galaxies, including some 300 AGN and some 1000 HII galaxies. Classification of Markarian galaxies provided Sy1 and Sy2 types and the definition of Starburst galaxies. Many new BCDG were discovered as well. The continuation of the FBS for stellar objects revealed FBS Blue Stellar Objects and FBS Late-type Stars, as well as optical identifications of IRAS sources have been carried out resulted in discovery of new ULIRGs and AGN. Markarian survey also led to many other objective prism surveys.

Markarian galaxies are reliable objects for MW studies of active galaxies, as they are bright enough and have been detected in all ranges of electromagnetic radiation; from $\gamma$-ray to radio. In one of the recent works, we have collected all available MW data from all-sky or large-area catalogs and have built MW SEDs for Markarian galaxies using 38 photometric points (Fermi, INTEGRAL, ROSAT, GALEX FUV/NUV, UBV, POSS I/II OjEN, SDSS ugriz, 2MASS JHK, WISE w1/w2/w3/w4, Spitzer IRAC/MIPS, AKARI 9/18/65/90/140/160 $\mu \mathrm{m}$, IRAS $12 / 25 / 60 / 100 \mu \mathrm{m}$, radio 4.85 and $1.4 \mathrm{GHz}, 843,612,326,152$ and 38 $\mathrm{MHz}$ ). These SEDs provide a possibility to group objects by their shapes and compare to existing physical properties to find various relations and refine the AGN classifications.

\section{Other projects based on FBS observing material}

As mentioned, as number of other projects were based on Markarian Survey: 
Table 5. Comparison of the main characteristics of large area spectroscopic surveys.

\begin{tabular}{|c|c|c|c|c|c|c|}
\hline Survey & Years & $\begin{array}{c}\text { Telescope } \\
\text { Equipment }\end{array}$ & $\begin{array}{c}\text { Sky area } \\
\text { Surface }\left(\operatorname{deg}^{2}\right)\end{array}$ & $\begin{array}{c}\text { Disp. } \mathbf{H} \gamma \\
\AA / \mathrm{mm}\end{array}$ & $\begin{array}{c}\text { Sp. range } \\
\AA\end{array}$ & $\mathbf{V}_{\text {lim }}$ \\
\hline FBS & 1965-1980 & $\begin{array}{c}\text { BAO } 1 \mathrm{~m} \text { Schmidt } \\
1.5 \text { prism }\end{array}$ & $\begin{array}{c}\delta>-15,|b|>15 \\
17,056\end{array}$ & 1800 & $3400-6900$ & 17.5 \\
\hline SBS & 1978-1991 & $\begin{array}{c}\text { BAO } 1 \mathrm{~m} \text { Schmidt } \\
1.5,3,4 \text { prisms }\end{array}$ & $\begin{array}{c}49<\delta<61,|b|>30 \\
965\end{array}$ & $1800 / 900 / 280$ & $3400-6950$ & 19.0 \\
\hline Case & 1983-1995 & $\begin{array}{c}\text { KPNO } 91 \mathrm{~cm} \\
1.8 \text { prism }\end{array}$ & $\delta>30,|b|>30$ & 1350 & $3400-5300$ & 18.0 \\
\hline HQS & $1985-1997$ & $\begin{array}{c}\text { CAHA } 81 \mathrm{~cm} \\
1.7 \text { prism }\end{array}$ & $\begin{array}{c}\delta>0,|b|>20 \\
14,000\end{array}$ & 1390 & $3400-5300$ & 19.0 \\
\hline HES & 1990-1996 & $\begin{array}{l}\text { ESO } 1 \mathrm{~m} \text { Schmidt } \\
4 \text { prism }\end{array}$ & $\begin{array}{c}\delta<2.5,|b|>30 \\
9,000\end{array}$ & 280 & $3400-5300$ & 18.0 \\
\hline SDSS & $2000-2021$ & $\begin{array}{c}\text { Apache Point } 2.5 \mathrm{~m} \\
\text { Double MOS }\end{array}$ & $\begin{array}{c}\delta>0,|b|>30 \\
14,555\end{array}$ & res. $2.5 \AA$ & $3000-10800$ & 22.0 \\
\hline
\end{tabular}

1) Second Byurakan Survey (SBS),

2) Second Part of the FBS: a) Search and studies for Blue Stellar Objects (BSO) and b) Search and studies for Late-Type Stars,

3) Optical Identifications of IRAS point sources (BIS and BIG objects, Byurakan-IR Stars and ByurakanIR Galaxies, respectively),

4) Search for asteroids on the DFBS.

Many papers have been produced based on these studies and a number of catalogues have been published in peer-reviewed journals and VizieR.

\section{Acknowledgements}

The work was supported by the Science Committee of RA, in the frames of the research project No. 21AG-1C053 "Revelation of the early stages of galaxy evolution by means of multiwavelength study of active galaxies".

\section{References}

Aharonian F., et al., 2005, Astron. Astrophys. , 437, 95

Ahumada R., et al., 2020, Astrophys. J. Suppl. Ser. , 249, 3

Ambartsumian V. A., 1958, in La structure et l'évolution de l'universe. pp 241-249

Antonucci R. R. J., Miller J. S., 1985, Astrophys. J. , 297, 621

Arakelian M. A., 1975, Communications of the Byurakan Astrophysical Observatory, 47, 3

Arakelyan M. A., Dibai É. A., Esipov V. F., 1973, Astrophysics, 9, 183

Arlen T., et al., 2013, Astrophys. J. , 762, 92

Baldwin J. A., Phillips M. M., Terlevich R., 1981, Publ. Astron. Soc. Pac. , 93, 5

Bicay M. D., Kojoian G., Seal J., Dickinson D. F., Malkan M. A., 1995, Astrophys. J. Suppl. Ser. , 98,369

Bird A. J., et al., 2010, Astrophys. J. Suppl. Ser. , 186, 1

Bolton J. G., Stanley G. J., Slee O. B., 1949, Nature. , 164, 101 
Carone T. E., et al., 1996, Astrophys. J. , 471, 737

Ciliegi P., Vignali C., Comastri A., Fiore F., La Franca F., Perola G. C., 2003, Mon. Not. R. Astron. Soc. , 342,575

Condon J. J., Cotton W. D., Greisen E. W., Yin Q. F., Perley R. A., Taylor G. B., Broderick J. J., 1998, Astron. J. , 115, 1693

Coziol R., Demers S., Pena M., Barneoud R., 1994, Astron. J. , 108, 405

Cutri R. M., et al. 2012, VizieR Online Data Catalog, p. II/311

Evans I. N., et al., 2010, Astrophys. J. Suppl. Ser. , 189, 37

Gigoyan K. S., Mickaelian A. M., Kostandyan G. R., 2019, Mon. Not. R. Astron. Soc. , 489, 2030

Gyulzadyan M. V., Mickaelian A. M., Abrahamyan H. V., Mikayelyan G. A., Paronyan G. M., 2018, Communications of the Byurakan Astrophysical Observatory, 65, 392

Haro G., 1956, Astron. J. , 61, 178

Harrison F. A., et al., 2013, Astrophys. J. , 770, 103

Heckman T. M., 1980, Astron. Astrophys. , 500, 187

Hoopes C. G., et al., 2007, Astrophys. J. Suppl. Ser. , 173, 441

Kandalyan R. A., Petrosyan A. R., 1989, Astrophysics, 30, 196

Kazarian M. A., Adibekyan V. Z., McLean B., Allen R. J., Petrosian A. R., 2010, Astrophysics, 53,57

Kojoian G., Sramek R. A., Dickinson D. F., Tovmassian H., Purton C. R., 1976, Astrophys. J. , 203, 323

Kojoian G., Chute P. A., Aumann C. E., 1984, Astron. J. , 89, 332

Korovyakovskii Y. P., Petrosyan A. R., Saakyan K. A., Khachikyan É. E., 1981, Astrophysics, 17,121

MacAlpine G. M., Feldman F. R., 1982, Astrophys. J. , 261, 412

Markarian B. E., 1963, Communications of the Byurakan Astrophysical Observatory, 34, 3

Markarian B. E., 1967, Astrofizika, 3, 24

Markarian B. E., Lipovetskii V. A., Stepanian D. A., 1983, Astrofizika, 19, 29

Markarian B. E., Erastova L. K., Lipovetsky V. A., Stepanian J. A., Shapovalova A. I., 1988, Astrofizika, 28,27

Markarian B. E., Lipovetsky V. A., Stepanian J. A., Erastova L. K., Shapovalova A. I., 1989, Soobshcheniya Spetsial'noj Astrofizicheskoj Observatorii, 62, 5

Markarian B. E., Lipovetsky V. A., Stepanian J. A., Erastova L. K., Shapovalova A. I., 1997, VizieR Online Data Catalog, p. VII/172 Massaro E., Mickaelian A. M., Nesci R., Weedman D., 2008, The Digitized First Byurakan Survey. ARACNE Editrice, Rome, 78 p.

Massaro E., Giommi P., Leto C., Marchegiani P., Maselli A., Perri M., Piranomonte S., Sclavi S., 2009, Astron. Astrophys. , 495, 691

Mazzarella J. M., Balzano V. A., 1986, Astrophys. J. Suppl. Ser. , 62, 751

McMahon R. G., Irwin M. J., Maddox S. J., 2000, VizieR Online Data Catalog, p. I/267

Mickaelian A. M., 1995, Astrophysics, 38, 349

Mickaelian A. M., 2000, Astronomical and Astrophysical Transactions, 18, 557

Mickaelian A. M., 2008, Astron. J. , 136, 946

Mickaelian A. M., Gigoyan K. S., 2006, Astron. Astrophys. , 455, 765

Mickaelian A. M., Sargsyan L. A., 2004, Astrophysics, 47, 213

Mickaelian A. M., Sargsyan L. A., 2010, Astrophysics, 53, 483

Mickaelian A. M., Gonçalves A. C., Véron-Cetty M. P., Véron P., 2001, Astrophysics, 44, 14

Mickaelian A. M., Hovhannisyan L. R., Engels D., Hagen H. J., Voges W., 2006, Astron. Astrophys. , 449, 425

Mickaelian A. M., et al., 2007, Astron. Astrophys. , 464, 1177

Mickaelian A. M., Abrahamyan H. V., Paronyan G. M., Harutyunyan G. S., 2013, Astronomische Nachrichten, 334,887

Mickaelian A. M., Abrahamyan H. V., Gyulzadyan M. V., Paronyan G. M., Mikayelyan G. A., 2018, Astrophys. Space. Sci. , 363, 237

Miyauchi-Isobe N., Maehara H., Nakajima K., 2010, Publications of the National Astronomical Observatory of Japan, 13 , 9

Nolan P. L., et al., 2012, Astrophys. J. Suppl. Ser. , 199, 31 
Osterbrock D. E., 1981, Astrophys. J. , 249, 462

Osterbrock D. E., Pogge R. W., 1985, Astrophys. J. , 297, 166

Pesch P., Sanduleak N., Stephenson C. B., 1991, Astrophys. J. Suppl. Ser. , 76, 1043

Petrosian A. R., Turatto M., 1986, Astron. and Astrophys. Suppl. Ser. , 65, 349

Petrosian A. R., Sahakian K. A., Khachikian E. Y., 1989, in Osterbrock D. E., Miller J. S., eds, Vol. 134, Active Galactic Nuclei. p. 445

Petrosian A., McLean B., Allen R. J., MacKenty J. W., 2007, Astrophys. J. Suppl. Ser. , 170, 33

Pustilnik S. A., et al., 2005, Astron. Astrophys. , 442, 109

Santos-Lleó M., et al., 2001, Astron. Astrophys. , 369, 57

Schmidt M., 1963, Nature. , 197, 1040

Schmitt J. L., 1968, Nature. , 218, 663

Seyfert C. K., 1943, Astrophys. J. , 97, 28

Stepanian J. A., 2005, Rev. Mex. Astron. Astrofis. , 41, 155

Tovmassian H. M., Terzian Y., 1974, Publ. Astron. Soc. Pac. , 86, 649

Ueda Y., Ishisaki Y., Takahashi T., Makishima K., Ohashi T., 2005, Astrophys. J. Suppl. Ser. , 161, 185

Veilleux S., Osterbrock D. E., 1987, Astrophys. J. Suppl. Ser. , 63, 295

Véron-Cetty M. P., Véron P., 2010, Astron. Astrophys. , 518, A10

Voges W., et al., 1999, Astron. Astrophys. , 349, 389

Voges W., et al., 2000, IAU Circ., 7432, 3

Weedman D. W., 1977, Vistas in Astronomy, 21, 55

Weedman D. W., Khachikyan E. E., 1968, Astrophysics, 4, 243

Woltjer L., 1959, Astrophys. J. , 130, 38

XMM 2010, XMM-Newton Serendipitous Source Catalogue: 2XMMi-DR3 XMM-Newton Survey Science Centre Consortium, XMM-SSC, Leicester, UK

Zickgraf F. J., Engels D., Hagen H. J., Reimers D., Voges W., 2003, Astron. Astrophys. , 406, 535

\section{Important papers on Markarian galaxies:}

\section{FBS lists (15)}

Markarian B.E. - Galaxies with an Ultraviolet Continuum // Ap, 3, 24-38, 1967

Markarian B.E. - Galaxies with Ultraviolet Continuum. II // Ap, 5, 206-225, 1969

Markarian B.E. - Galaxies with ultraviolet continuum. III // Ap, 5, 286-301, 1969

Markarian, B. E.; Lipovetskii, V. A. -Galaxies with ultraviolet continuum. IV // Ap, 7, 299-310, 1971

Markarian, B. E.; Lipovetskii, V. A. - Galaxies with ultraviolet continuum. V // Ap, 8, 89-99, 1972

Markarian, B. E.; Lipovetskii, V. A. - Galaxies with ultraviolet continuum. VI // Ap, 9, 283-295, 1973

Markarian, B. E.; Lipovetskii, V. A. - Galaxies with ultraviolet continuum. VII // Ap, 10, 185-194, 1974

Markarian, B. E.; Lipovetskii, V. A. - Galaxies with ultraviolet continuum. VIII // Ap, 12, 241-254, 1976

Markarian, B. E.; Lipovetskii, V. A. - Galaxies with ultraviolet continuum. IX // Ap, 12, 429-442, 1976

Markarian, B. E.; Lipovetskii, V. A.; Stepanian, J. A. - Galaxies with ultraviolet continuum. X // Ap, 13, 116-125, 1977

Markarian, B. E.; Lipovetskii, V. A.; Stepanian, J. A. - Galaxies with ultraviolet continuum. XI // Ap, 13, 215-228, 1977

Markarian, B. E.; Lipovetskii, V. A.; Stepanian, J. A. - Galaxies with ultraviolet continuum. XII // Ap, 15, 130-141, 1979

Markarian, B. E.; Lipovetskii, V. A.; Stepanian, J. A. - Galaxies with ultraviolet continuum. XIII // Ap, 15, 235-250, 1980

Markarian, B. E.; Lipovetskii, V. A.; Stepanian, J. A. - Galaxies with ultraviolet continuum. XIV // Ap, 15, 363-376, 1980

Markarian, B. E.; Lipovetskii, V. A.; Stepanian, J. A. - Galaxies with ultraviolet continuum. XV // Ap, 17, 321-332, 1982

\section{FBS catalogs (5)}

Balzano \& Mazzarella 1986

Markarian, B. E.; Lipovetsky, V. A.; Stepanian, J. A.; Erastova, L. K.; Shapovalova, A. I. - The First Byurakan Survey - a Catalogue of Galaxies with Ultraviolet Continuum // Com. SAO, 62, 5-121, 1989 (in Russian)

Markarian B.E. - Catalogue of Markarian Galaxies // VizieR On-line Data Catalog, VII/61A, 1994

Markarian, B. E.; Lipovetsky, V. A.; Stepanian, J. A.; Erastova, L. K.; Shapovalova, A. I. - First Byurakan Survey (FBS) // VizieR On-line Data Catalog, VII/172, 1997

Petrosian, A., McLean, B., Allen, R. J., MacKenty, J. W., ApJS 170, 33, 2007 


\section{Spectra of Markarian galaxies (9+)}

Arakelian, M. A.; Dibay, Eh. A.; Esipov, V. F.; Markarian, B. E. - Spectra of Markarian galaxies. II // Ap, 6, 189-194, 1970 Arakelian, M. A.; Dibay, Eh. A.; Esipov, V. F.; Markarian, B. E. - Spectra of Markarian galaxies. III // Ap, 7, 102-108, 1971 Markarian, B. E.; Lipovetskii, V. A.; Stepanian, J. A. - Spectra of galaxies with ultraviolet continuum. I // Ap, 16, 1-7, 1980 Markarian, B. E.; Lipovetskii, V. A.; Stepanian, J. A. - Spectra of galaxies with ultraviolet continuum. II // Ap, 16, 353-359, 1981 Markarian, B. E.; Lipovetskii, V. A.; Stepanian, J. A. - Spectra of galaxies with ultraviolet continuum. III // Ap, 19, 129-133, 1983 Markarian, B. E.; Lipovetskii, V. A.; Stepanian, J. A. - Spectra of galaxies with ultraviolet continuum. IV // Ap, 21, 581-587, 1985

Markarian, B. E.; Erastova, L. K.; Lipovetskii, V. A.; Stepanian, J. A.; Shapovalova, A. I. - Spectra of galaxies with ultraviolet continuum. V // Ap, 22, 127-135, 1985

Markarian, B. E.; Erastova, L. K.; Lipovetskii, V. A.; Stepanian, J. A.; Shapovalova, A. I. - Spectra of galaxies with Ultraviolet Continuum. VI // Ap, 28, 14-21, 1988

Markarian, B. E.; Erastova, L. K.; Lipovetskii, V. A.; Stepanian, J. A.; Shapovalova, A. I. - Spectra of galaxies with Ultraviolet Continuum. VII // Ap, 28, 283-288, 1988

Khachikian/Weedman papers

\section{FBS Seyferts (12)}

Arakelian, M. A.; Dibay, Eh. A.; Esipov, V. F.; Markarian, B. E. - New Seyfert-type objects in the second Markarian's list // Astron. Circular, 568, 1-2, 1970 (in Russian)

Arakelian, M. A.; Dibay, Eh. A.; Esipov, V. F.; Markarian, B. E. - New Seyfert-type objects in the second and third Markarian's lists // Astron. Circular, 586, 1-3, 1970 (in Russian)

Arakelian, M. A.; Dibay, Eh. A.; Esipov, V. F.; Markarian, B. E. - New Seyfert-type objects in the third Markarian's list // Astron. Circular, 568, 2-4, 1970 (in Russian)

Markarian, B. E.; Lipovetskii, V. A.; Stepanian, J. A. - New Seyfert-type objects in list XII of galaxies with ultraviolet continuum // Astron. Letters, 5, 269-271, 1979

Markarian, B. E.; Lipovetskii, V. A.; Stepanian, J. A. - Seyfert-Type Objects from the 13th and 14th Lists of Galaxies with UVContinuum // Astron. Circular, 1134, 6-8, 1980 (in Russian)

Afanasiev, V. L.; Lipovetskii, V. A.; Markarian, B. E.; Stepanian, J. A. - New Seyfert-Type Objects from Observations on BTA // Astron. Circular, 1039, 1-2, 1979 (in Russian)

Afanasiev, V. L.; Lipovetskii, V. A.; Markarian, B. E.; Stepanian, J. A. - Observations with the 6-m telescope of galaxies with ultraviolet continuum of Seyfert type // Ap, 16, 119-127, 1980

Markarian, B. E.; Stepanian, J. A.; Lipovetskii, V. A. - New Seyfert-Type Objects from the Last Lists of Galaxies with UV-Continuum // Astron. Circular, 1125, 4-6, 1980 (in Russian)

Markarian, B. E.; Stepanian, J. A.; Lipovetskii, V. A. - Faint Seyfert I Type Galaxies // Astron. Circular, 1168, 2-5, 1981 (in Russian) Markarian, B. E.; Stepanian, J. A.; Lipovetskii, V. A. - Two New Remarkable Seyfert-Type Galaxies // Astron. Circular, 1233, 1-2, 1982 (in Russian)

Markarian, B. E.; Stepanian, J. A.; Lipovetskii, V. A. - Two New Remarkable Seyfert-Type Galaxies // Astron. Circular, 1237, 1, 1982 (in Russian)

Markarian, B. E.; Stepanian, J.; Lipovetski, V. I.; Shapovalova, A. I. - New Seyfert Galaxies from the First Byurakan Sky Survey // Astron. Circular, 1428, 7-8, 1986 (in Russian)

\section{Markarian galaxies nature (4)}

Markarian B.E. - On the nature of galaxies with ultraviolet continuum. I. Principal spectral and colour characteristics // Ap, 8, 100-105, 1972

Markarian B.E. - On the nature of galaxies with ultraviolet continuum: II. Objects with broad emission lines // Ap, 9, 1-8, 1973

Markarian, B. E.; Stepanian, J. A. - On the nature of galaxies with ultraviolet continuum. III. Surface brightness, morphology, and activity // Ap, 13, 372-379, 1977

Markarian B.E. - On the nature of galaxies with UV continuum having broad emission lines // A\&A, 58, 139-143, 1977

\section{FBS bright QSOs (2)}

Markarian, B. E.; Stepanian, J. A.; Lipovetskii, V. A. - Bright Quasar Search and the FBS Continuation // Com. SAO, 61, 88-91, 1988 (in Russian)

Markarian, B. E.; Stepanian, J. A.; Lipovetskii, V. A. - Bright Quasar Search and the FBS Continuation // Proc. Conf.: QSO Physical Properties and Spectra, held at Special Astrophys. Obs., Moscow, 8-11 Sep 1987, Moscow, p. 88 , 1989 (in Russian)

\section{FBS general (3)}

Markarian B.E. - Galaxies with the ultraviolet continuum // Proc. IAU S29: Non-stable Phenomena in Galaxies, Byurakan, May 4-12, 1966. Publ. House Acad. Sci. Arm. SSR Yerevan, p. 99-103, 1968 (in Russian)

Markarian, B. E.; Lipovetskii, V. A.; Stepanian, J. A. - Galaxies with UV-Continuum from Observations on BTA // Proc. Borjomi Meeting, 1981 (in Russian)

Lipovetsky, V. A.; Markarian, B. E.; Stepanian, J. A. - The First Byurakan Survey (FBS) and Search Problems for AGN // Proc. IAU S121: Observational Evidence of Activity in Galaxies, held in Byurakan, Armenia, USSR, 3-7 June 1986. Eds. E.Ye. Khachikian, K.J. Fricke \& J. Melnick. Kluwer Academic Publishers, Dordrecht, p. 17, 1987

\section{Markarian webpage created by A. M. Mickaelian and G. A. Mikayelyan in 2013: http: // markarian. aras.am/}

